\title{
PSEUDOCHARACTERS OF BRAID GROUPS AND PRIME LINKS
}

\author{
A. V. MALYUTIN
}

\begin{abstract}
Pseudocharacters of groups have recently found an application in the theory of classical knots and links in $\mathbb{R}^{3}$. More precisely, there is a relationship between pseudocharacters of Artin's braid groups and the properties of links represented by braids. In the paper, this relationship is investigated and the notion of kernel pseudocharacters of braid groups is introduced. It is proved that if a kernel pseudocharacter $\phi$ and a braid $\beta$ satisfy $|\phi(\beta)|>C_{\phi}$, where $C_{\phi}$ is the defect of $\phi$, then $\beta$ represents a prime link (i.e., a link that is noncomposite, nonsplit, and nontrivial). Furthermore, the space of braid group pseudocharacters is studied and a way is described to obtain nontrivial kernel pseudocharacters from an arbitrary braid group pseudocharacter that is not a homomorphism. This makes it possible to employ an arbitrary nontrivial braid group pseudocharacter for the recognition of prime knots and links.
\end{abstract}

\section{INTRODUCTION}

A function $\varphi: G \rightarrow \mathbb{R}$ on a group $G$ is called a pseudocharacter if the following conditions are fulfilled:

$$
C_{\varphi}:=\sup _{g_{1}, g_{2} \in G}\left|\varphi\left(g_{1} g_{2}\right)-\varphi\left(g_{1}\right)-\varphi\left(g_{2}\right)\right|<\infty
$$

and

$$
\forall z \in \mathbb{Z}, g \in G: \varphi\left(g^{z}\right)=z \varphi(g) .
$$

The quantity $C_{\varphi}$ is called the defect of $\varphi$. The simplest examples of pseudocharacters are real-valued homomorphisms. Their set coincides with the set of pseudocharacters with defect 0 .

If a function satisfies condition (11), then it is called a quasicharacter or quasimorphism. Pseudocharacters are sometimes also referred to as homogeneous quasimorphisms.

Pseudocharacters and quasicharacters of groups have recently been intensively studied. It has turned out that these objects are useful for the study of classical knots and links in $\mathbb{R}^{3}$. The relationship between quasimorphisms and knots emerges from braid theory when we consider quasimorphisms of Artin's braid groups $B_{2}, B_{3}, B_{4}, \ldots$ and the standard way of representing knots and links in the form of geometric braids. (See a brief description of this representation in $\S 1$ below.) For example, it was discovered that certain link invariants are quasimorphisms of braid groups (see [12, 2]).

In this paper, we treat another type of dependence between braid group quasimorphisms and knots. This dependence was introduced in [15, where a braid group pseudocharacter called a twist number was defined. The twist number is not a link invariant

2000 Mathematics Subject Classification. Primary 20F36.

Key words and phrases. Knot, link, braid, pseudocharacter, quasimorphism.

Supported in part by RFBR (grant 08-01-00379a) and the RF President Program for Support of leading Scientific Schools (grant NSh-2460.2008.1112). 
(i. e., there are braids representing one and the same link type and having distinct twist numbers). Nevertheless, the twist number of a braid provides certain information about the corresponding link. For example, if the absolute value of the twist number of a braid is greater than 1, then the corresponding link is prime. It turns out that other pseudocharacters of the braid group detect prime links in a similar way. These pseudocharacters are said to be kernel. In $\S 2$ below, we define the kernel pseudocharacters and present the following theorem.

Theorem 1. Suppose $n \geq 3$ and $\varphi: B_{n} \rightarrow \mathbb{R}$ is a kernel pseudocharacter with defect $C_{\varphi}$. Assume that $\beta \in B_{n}$. If $|\varphi(\beta)|>C_{\varphi}$, then $\beta$ represents a prime link (i.e., a link that is noncomposite, nonsplit, and nontrivial). In particular, if $\beta$ represents a knot, then this knot is prime.

The definitions imply that the pseudocharacters of a group form a real vector space. (For a group $G$, we denote this space by $\mathcal{P X}(G)$.) The kernel pseudocharacters form a linear subspace in the space of braid group pseudocharacters. If $n \geq 3$, then the space $\mathcal{P X}\left(B_{n}\right)$ of pseudocharacters of the braid group $B_{n}$ is infinite-dimensional 1 (This fact follows, for instance, from the results of 3.) Nevertheless, finding a specific pseudocharacter of the braid group and an algorithm for its computation is a hard and interesting problem. Only a few series of braid group pseudocharacters have been described explicitly up to now (see [12, 15]). The problem of finding new kernel pseudocharacters seems to be even more difficult. In the present paper, we reduce this problem to finding arbitrary pseudocharacters. More precisely, we present a combinatorial formula that describes a specific projection of the space of all pseudocharacters of the braid group onto the subspace of kernel pseudocharacters. The formula automatically converts any algorithm that computes a pseudocharacter of the braid group into an algorithm that computes the kernel pseudocharacter arising under this projection. The fact that our formula actually determines a projection of the space of all braid group pseudocharacters onto the subspace of kernel pseudocharacters is stated in Theorem 2 (see $\S 3$ ). The proof of Theorem 2 occupies most of the paper.

The considerations that allowed us to find the combinatorial formula mentioned above deserve special attention. They may be outlined as follows. We observe that each map $f: G \rightarrow \mathbb{R} H$ from a group $G$ to the group ring $\mathbb{R} H$ of a group $H$ has an associated linear operator $f^{*}$ from the space of functions on $H$ to the space of functions on $G$. If the image $f^{*}(\mathcal{P X}(H))$ is contained in $\mathcal{P} \mathcal{X}(G)$, we say that $f$ is pseudohomomorphic. (We easily see that the simplest examples of pseudohomomorphic mappings are linear compositions of homomorphisms.) The pseudohomomorphic maps are of interest, in particular, in light of the above problem of describing pseudocharacters of the braid group and finding algorithms for their computation, because pseudohomomorphic maps transform known pseudocharacters into new ones. Using a geometric interpretation of braids, we can construct various pseudohomomorphic maps for braid groups. By a combination of such maps, it is possible to find a pseudohomomorphic map that determines a projection with the required properties. We denote this projection by $\mathbb{T}$. A more detailed discussion of pseudohomomorphic maps for braid groups will appear in a forthcoming publication. In this paper we only present a pseudohomomorphic map that yields the projection $\mathbb{T}$ and prove Theorem 2 . (In fact, that theorem states that our map is indeed pseudohomomorphic and $\mathbb{T}$ is a projection onto the subspace of kernel pseudocharacters.)

\footnotetext{
${ }^{1}$ The space $\mathcal{P X}\left(B_{2}\right)$ is one-dimensional. Indeed, $B_{2}$ is isomorphic to $\mathbb{Z}$, while the definition of a pseudocharacter implies immediately that each pseudocharacter of $\mathbb{Z}$ is a homomorphism. It follows that $\mathcal{P} \mathcal{X}\left(B_{2}\right) \cong \mathcal{P} \mathcal{X}(\mathbb{Z}) \cong \mathbb{R}$.
} 
It should be noted that our results allow us to use an arbitrary braid group pseudocharacter that is not a homomorphism 2 for recognizing prime knots and links. The explanation is as follows. The "combinatorial formula" that determines the projection $\mathbb{T}$ for the space of pseudocharacters of the braid group is actually a series of homogeneous formulas for the series of projections $\mathbb{T}_{2}, \mathbb{T}_{3}, \mathbb{T}_{4}, \ldots$ corresponding to the series $\mathcal{P X}\left(B_{2}\right)$, $\mathcal{P X}\left(B_{3}\right), \mathcal{P X}\left(B_{4}\right), \ldots$ of spaces of pseudocharacters for the braid groups $B_{2}, B_{3}, B_{4}, \ldots$ We recall that braid groups of smaller indices can be regarded as subgroups in braid groups of larger indices. If $\psi: B_{n} \rightarrow \mathbb{R}$ is a pseudocharacter, then the restrictions $\psi_{n-1}, \psi_{n-2}, \ldots$ of $\psi$ to the subgroups $B_{n-1}, B_{n-2}, \ldots$ are pseudocharacters of these subgroups. It turns out that if $\psi$ is not a homomorphism, then at least one of the kernel pseudocharacters $\mathbb{T}_{n}(\psi), \mathbb{T}_{n-1}\left(\psi_{n-1}\right), \ldots$ is nontrivial. Hence, we obtain a sufficient condition for prime links represented by elements of the corresponding braid group.

Structure of the paper: $\S 1$ contains the basic definitions from braid group theory and a description of the standard map from a braid group to the set of links in $\mathbb{R}^{3}$.

In $\S 2$, we introduce the notion of a kernel pseudocharacter and prove Theorem 1.

In $\S 3$ we present the combinatorial formula mentioned above, which determines the projection $\mathbb{T}$ of the space of pseudocharacters for the braid group onto the subspace of kernel pseudocharacters. In that section, we also formulate Theorem 2.

The subsequent sections are devoted to the proof of Theorem 2.

In $\S 4$, we prove two lemmas about pseudocharacters of a normal subgroup of finite index in a group. We apply these lemmas to the case of the braid group $B_{n}$ and its subgroup $P_{n}$ of pure braids ( $P_{n}$ is a normal subgroup of finite index in $\left.B_{n}\right)$.

In $\S 5$, a notion of a strand disentangling procedure for braids is introduced. These procedures are maps $B_{n} \rightarrow B_{n}$. They have clear geometrical meaning. The restrictions of these maps to the subgroup $P_{n}$ of pure braids yield a family of endomorphisms REL ${ }_{J}$ : $P_{n} \rightarrow P_{n}, J \subset\{1, \ldots, n\}$. We use these endomorphisms to construct a combinatorial formula for our projection $\mathbb{T}$ in $\S 3$.

In $\S 6$, we study the endomorphisms REL $J: P_{n} \rightarrow P_{n}$ defined in $\S 5$.

In $\S 7$, the alternating sum lemma for endomorphisms $\mathrm{REL}_{J}$ is proved.

In $\S 8$, we prove a proposition concerning certain endomorphisms of the space $\mathcal{P X}\left(B_{n}\right)$ that are related to the endomorphisms REL . $_{\text {. }}$

Finally, in $\S 9$ we derive Theorem 2 from the results of the preceding sections.

\section{§1. BRaid groups AND THE USUAL REPRESEnTAtion OF LINKS BY BRAIDS}

1.1. The braid group. The Artin braid group of rank $n$ is determined by the presentation

$$
B_{n}:=\left\langle\sigma_{1}, \ldots, \sigma_{n-1}\left|\sigma_{i} \sigma_{j}=\sigma_{j} \sigma_{i},\right| i-j \mid \geq 2 ; \sigma_{i} \sigma_{i+1} \sigma_{i}=\sigma_{i+1} \sigma_{i} \sigma_{i+1}\right\rangle .
$$

The group $B_{1}$ is trivial. The elements of the groups $B_{1}, B_{2}, B_{3}, \ldots$ are called braids. The generators $\sigma_{i}$ are Artin's generators. By a braid group we usually mean a braid group with a fixed set of Artin's generators.

If $n$ is fixed and $k<n$, then we denote by $\bar{B}_{k}$ the subgroup of $B_{n}$ generated by the elements $\sigma_{1}, \ldots, \sigma_{k-1}$. (Clearly, we have $\bar{B}_{k} \cong B_{k}$.) By the canonical monomorphism from $B_{k}$ to $B_{n}$ we mean the monomorphism sending the generator $\sigma_{i} \in B_{k}$ to the generator $\sigma_{i} \in B_{n}$. (Therefore, $\bar{B}_{k}$ is the image of $B_{k}$ under the canonical monomorphism.)

\footnotetext{
${ }^{2}$ Recall that for $n \geq 2$ the braid group $B_{n}$ admits a unique (up to multiplication by a constant) nontrivial real-valued homomorphism. In other words, the subspace of real-valued homomorphisms of $B_{n}$ with $n \geq 2$ is a one-dimensional subspace in $\mathcal{P X}\left(B_{n}\right)$.
} 
1.2. Pure braids. Let $\Sigma_{n}$ be the symmetric group on the set $\{1, \ldots, n\}$. It is well known that the map $\left\{\sigma_{1}, \ldots, \sigma_{n-1}\right\} \rightarrow \Sigma_{n}$ sending $\sigma_{i}$ to the permutation $(i, i+1)$ extends to an epimorphism $B_{n} \rightarrow \Sigma_{n}$. The kernel of the epimorphism is the pure braid group $P_{n}$. The group $P_{n}$ is a normal subgroup of index $n$ ! in $B_{n}$. We have the following exact sequence:

$$
1 \rightarrow P_{n} \rightarrow B_{n} \rightarrow \Sigma_{n} \rightarrow 1
$$

The braids in $P_{n}$ are said to be pure. Since $P_{n}$ has index $n$ ! in $B_{n}$, it follows that the braid $\beta^{n !}$ is pure for each $\beta \in B_{n}$.

If $n$ is fixed and $k<n$, then we denote by $\bar{P}_{k}$ the subgroup $\bar{B}_{k} \cap P_{n}$ of $B_{n}$. Obviously, the subgroup $\bar{P}_{k}$ is the image of the subgroup $P_{k} \subset B_{k}$ under the canonical monomorphism $B_{k} \rightarrow B_{n}$.

We introduce the following notation: for $i$ and $j$ with $1 \leq i<j \leq n$, we set

$$
a_{i j}:=\sigma_{i} \sigma_{i+1} \cdots \sigma_{j-2} \sigma_{j-1}^{2} \sigma_{j-2}^{-1} \cdots \sigma_{i+1}^{-1} \sigma_{i}^{-1} \in B_{n} .
$$

It is known that, in $B_{n}$, the set $\left\{a_{i j}, 1 \leq i<j \leq n\right\}$ generates $P_{n}$, while the set $\left\{a_{i j}, 1 \leq i<j \leq k\right\}$ generates $\bar{P}_{k}$ (see, e.g., [17). The elements $a_{i j}$ will be called the Markov generators.

1.3. Representation of links by braids. The standard geometric interpretation of braids (for a detailed description, see, e.g., 7]) allows us to use braids for presentation of knots and links. We briefly describe the classical procedure that sends a braid $\beta \in B_{n}$ to a link $L(\beta)$ in $\mathbb{R}^{3}$. Let $W=w_{1} w_{2} \cdots w_{s}$ be a word over Artin generators and their
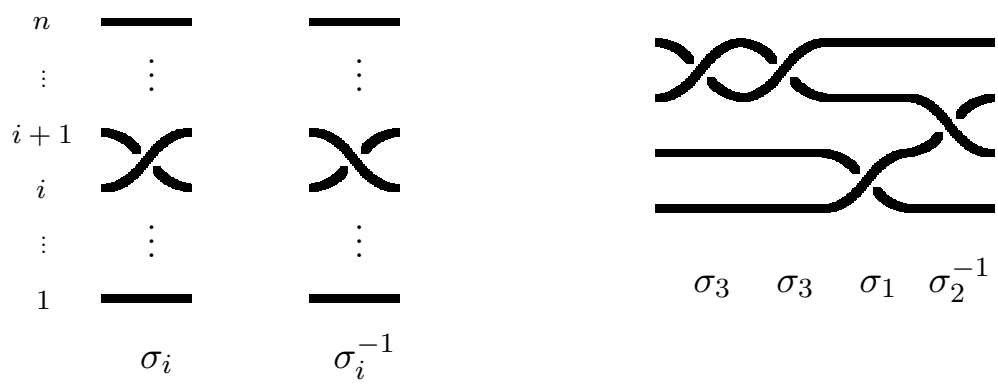

FIGURE 1. The diagrams $\mathcal{D}_{\sigma_{i}}^{\langle n\rangle}, \mathcal{D}_{\sigma_{i}^{-1}}^{\langle n\rangle}$, and $\mathcal{D}_{\sigma_{3} \sigma_{3} \sigma_{1} \sigma_{2}^{-1}}^{\langle 4\rangle}$.

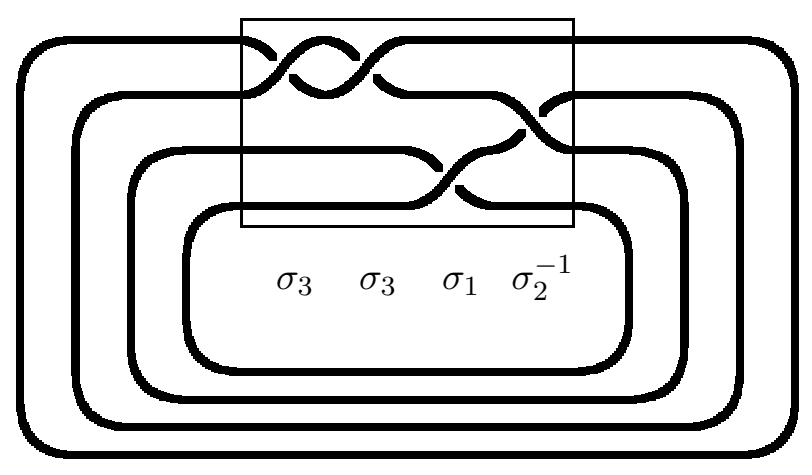

FiguRE 2. The diagram $\overline{\mathcal{D}}_{\sigma_{3} \sigma_{3} \sigma_{1} \sigma_{2}^{-1}}^{\langle 4\rangle}$. 
inverses that represents $\beta$. For $i \in\{1, \ldots, n\}$, let the plane diagram $\mathcal{D}_{\sigma_{i}}^{\langle n\rangle}$ represent the symbol $\sigma_{i}$, and let the diagram $\mathcal{D}_{\sigma_{i}^{-1}}^{\langle n\rangle}$ represent $\sigma_{i}^{-1}$ (see Figure 1). We define the diagram $\mathcal{D}_{W}^{\langle n\rangle}$ as the concatenation of the diagrams $\mathcal{D}_{w_{1}}^{\langle n\rangle}, \mathcal{D}_{w_{2}}^{\langle n\rangle}, \ldots, \mathcal{D}_{w_{s}}^{\langle n\rangle}$. Figure 11 shows the diagram $\mathcal{D}_{\sigma_{3} \sigma_{3} \sigma_{1} \sigma_{2}^{-1}}^{\langle 4\rangle}$ as an example.

To obtain a link, we "close" our diagram $\mathcal{D}_{W}^{\langle n\rangle}$ in the way depicted in Figure 2 , The resulting diagram $\overline{\mathcal{D}}_{W}^{\langle n\rangle}$ is the standard projection for a certain link in $\mathbb{R}^{3}$. It is well known that the link type $L_{W}$ of this link is uniquely determined by $\beta$; i.e., it does not depend on the word $W$ representing $\beta$. We set $L(\beta):=L_{W}$ and say that $\beta$ represents $L(\beta)$.

It is known that conjugate braids represent one and the same link type. By the Alexander theorem [1, for each link type $L$ there exists a braid $\beta$ such that $L=L(\beta)$.

\section{§2. KERNEL PSEUdocharaCters of BRAID GROUPS AND PRIME LINKS}

In this section, we introduce the notion of a kernel pseudocharacter and prove Theorem 1.

2.1. Definition: kernel pseudocharacters. A pseudocharacter $\varphi: B_{n} \rightarrow \mathbb{R}$ of the braid group $B_{n}$ is said to be a kernel if $\varphi$ maps $\bar{B}_{n-1}$ to zero. (We recall that $\bar{B}_{n-1}$ is the subgroup generated by $\sigma_{1}, \ldots, \sigma_{n-2}$.)

2.2. Remark. Our choice of the term "kernel" is explained by the following construction. We easily see that for each homomorphism $f: G \rightarrow H$ and each pseudocharacter $\varphi: H \rightarrow \mathbb{R}$ the composition $\varphi \circ f: G \rightarrow \mathbb{R}$ is a pseudocharacter. In terms of category theory, this means that the functor that sends a group to the space of pseudocharacters of that group is contravariant: a group homomorphism $f: G \rightarrow H$ induces a linear operator $f^{*}: \mathcal{P X}(H) \rightarrow \mathcal{P X}(G)$. Consider the linear operator $i^{*}: \mathcal{P X}\left(B_{n}\right) \rightarrow \mathcal{P X}\left(B_{n-1}\right)$ induced by the canonical monomorphism $i: B_{n-1} \rightarrow B_{n}$. By definition, the set of kernel pseudocharacters of $B_{n}$ is the kernel of $i^{*}$. Therefore, the kernel pseudocharacters constitute a linear subspace in $\mathcal{P X}\left(B_{n}\right)$ (the kernel $\operatorname{ker}\left(i^{*}\right)$ ). This subspace is called the space of kernel pseudocharacters of $B_{n}$.

2.3. Remark. It is easily seen that the subspace $\mathcal{K}\left(B_{3}\right)$ of kernel pseudocharacters in $\mathcal{P X}\left(B_{3}\right)$ has codimension 1. Since $\mathcal{P X}\left(B_{3}\right)$ is infinite-dimensional (as was mentioned in the Introduction), it follows that so is $\mathcal{K}\left(B_{3}\right)$. It is natural to conjecture that the space of kernel pseudocharacters of $B_{n}$ is also infinite-dimensional if $n \geq 4$, but the existence of nontrivial kernel pseudocharacters in $\mathcal{P X}\left(B_{n}\right)$ with $n \geq 4$ is nonobvious a priori. The results of [15], where a series of kernel pseudocharacters was described, show that the space of kernel pseudocharacters for $B_{n}$ with $n \geq 4$ is nontrivial.

2.4. Remark. The definition of kernel pseudocharacters involves the subgroup $\bar{B}_{n-1}$. This means that the definition implicitly involves the Artin generators. (As mentioned in $\S 1$, the braid group is usually equipped with a fixed set of Artin generators.) Note that the property for a pseudocharacter to be a kernel does not depend on a specific set of Artin generators. This fact follows immediately from the description of the automorphism group of the braid group (see [8]).

2.5. Remark. A braid $\beta \in B_{n}$ is said to be split if there is a braid $\alpha$ such that the braid $\beta^{\prime}=\alpha \beta \alpha^{-1}$ belongs to the subgroup generated by the set $\left\{\sigma_{1}, \ldots, \sigma_{n-1}\right\} \backslash\left\{\sigma_{r}\right\}$ for some $r \in\{1, \ldots, n-1\}$. It is easily seen that a pseudocharacter is kernel if and only if it sends each split braid to zero.

Theorem 1. Suppose $n \geq 3$ and $\varphi: B_{n} \rightarrow \mathbb{R}$ is a kernel pseudocharacter with defect $C_{\varphi}$. Let $\beta \in B_{n}$. If $|\varphi(\beta)|>C_{\varphi}$, then $\beta$ represents a prime link (i.e., a link that is 
noncomposite, nonsplit, and nontrivial). In particular, if $\beta$ represents a knot, then the knot is prime.

Proof. We argue by contradiction. Assume that the link represented by $\beta$ is not prime. Then [16. Proposition 6.1] implies that the conjugacy class of $\beta$ contains a braid $\beta^{\prime}=$ $\sigma_{n-1} \alpha_{1} \sigma_{n-1}^{-1} \alpha_{2}$ such that the braids $\alpha_{1}$ and $\alpha_{2}$ belong to the subgroup $\bar{B}_{n-1}$. (The proposition follows from the results of [4, 5, 6, 9] concerning braid representations of trivial, split, and composite links.) It is well known that each pseudocharacter is conjugacy invariant (see, e.g., [10]). Consequently, we have

$$
\begin{aligned}
|\varphi(\beta)|=\left|\varphi\left(\beta^{\prime}\right)\right|=\left|\varphi\left(\sigma_{n-1} \alpha_{1} \sigma_{n-1}^{-1} \alpha_{2}\right)\right| & \leq\left|\varphi\left(\sigma_{n-1} \alpha_{1} \sigma_{n-1}^{-1}\right)\right|+\left|\varphi\left(\alpha_{2}\right)\right|+C_{\varphi} \\
& =\left|\varphi\left(\alpha_{1}\right)\right|+\left|\varphi\left(\alpha_{2}\right)\right|+C_{\varphi}=C_{\varphi},
\end{aligned}
$$

a contradiction.

\section{§3. Combinatorial formula for a projection onto the subspace OF KERNEL PSEUDOCHARACTERS}

In this section, we present a combinatorial formula that determines a projection of the space $\mathcal{P X}\left(B_{n}\right)$ of pseudocharacters of the braid group onto the subspace of kernel pseudocharacters.

The formula involves a family of endomorphisms of the pure braid group $P_{n}$. We denote these endomorphisms by $\mathrm{REL}_{J}: P_{n} \rightarrow P_{n}, J \subset\{1, \ldots, n\}$. (The family contains $2^{n}$ endomorphisms, because $2^{n}$ is the number of subsets of $\{1, \ldots, n\}$.) We define the homomorphisms REL $\mathrm{R}_{J}$ in the following existence and uniqueness statement, which will be proved in $\S 6$ below.

3.1. Proposition. Let $P_{n}$ be the pure braid group with the Markov generators $\left\{a_{i j}\right.$, $1 \leq i<j \leq n\}$. Then each subset $J \subset\{1, \ldots, n\}$ determines a unique homomorphism $\mathrm{REL}_{J}: P_{n} \rightarrow P_{n}$ satisfying the following condition:

for any $i<j \in\{1, \ldots, n\}$, we have

$$
\operatorname{REL}_{J}\left(a_{i j}\right)= \begin{cases}1 & \text { if } i \in J \text { or } j \in J, \\ a_{i j} & \text { if }\{i, j\} \cap J=\varnothing .\end{cases}
$$

3.2. Definition: the projection operator $\mathbb{T}$. For a pseudocharacter $\phi: B_{n} \rightarrow \mathbb{R}$, we define a function $\mathbb{T}_{\phi}: B_{n} \rightarrow \mathbb{R}$ as follows:

$$
\mathbb{T}_{\phi}(\beta):=\sum_{J \subset\{1, \ldots, n\}} \frac{(-1)^{|J|} \cdot \phi\left(\operatorname{REL}_{J}\left(\beta^{n !}\right)\right)}{n !} .
$$

Here, $\beta \in B_{n}$ is an arbitrary braid, and the sum is taken over all subsets of $\{1, \ldots, n\}$. The braids $\operatorname{REL}_{J}\left(\beta^{n !}\right)$ are well defined because the braid $\beta^{n !}$ is pure.

\section{Theorem 2.}

1. For each pseudocharacter $\phi \in \mathcal{P X}\left(B_{n}\right)$, the function $\mathbb{T}_{\phi}: B_{n} \rightarrow \mathbb{R}$ determined by formula $(*)$ is a kernel pseudocharacter.

2. For each kernel pseudocharacter $\psi \in \mathcal{P X}\left(B_{n}\right)$, we have $\mathbb{T}_{\psi}=\psi$.

We denote by $\mathbb{T}_{n}$ the endomorphism of $\mathcal{P X}\left(B_{n}\right)$ that sends a pseudocharacter $\phi$ to the pseudocharacter $\mathbb{T}_{\phi}$. We abbreviate $\mathbb{T}_{n}$ to $\mathbb{T}$ if this does not lead to confusion. The form of formula $(*)$ obviously implies that $\mathbb{T}$ is a linear operator. Theorem 2 says that the image of $\mathbb{T}$ is the subspace of kernel pseudocharacters, and the restriction of $\mathbb{T}$ to the subspace of kernel pseudocharacters is the identity map. In other words, $\mathbb{T}$ is a projection of the space of all braid group pseudocharacters onto the subspace of kernel pseudocharacters. 
3.3. Remarks on algorithms. 1. Suppose that we have a computational algorithm for a certain pseudocharacter $\phi \in \mathcal{P X}\left(B_{n}\right)$. Then, obviously, using formula (*) we can compute the values of the kernel pseudocharacter $\mathbb{T}_{\phi}=\mathbb{T}_{n}(\phi)$. To compute $\mathbb{T}_{\phi}(\beta)$ directly by our formula, we must find the braids $\operatorname{REL}_{J}\left(\beta^{n !}\right)$ for $J \subset\{1, \ldots, n\}$. In their turn, to compute these braids directly by using the above definition of the homomorphisms REL , $_{J}$ we find a representation of the braid $\beta^{n !}$ over the Markov generators. If the braid $\beta$ is initially written in the Artin generators, the latter procedure can be very tiresome. It turns out, however, that we can easily obtain a word $W$ (in the Artin generators) for $\operatorname{REL}_{J}\left(\beta^{n !}\right)$ from a word $V$ in the Artin generators for $\beta^{n !}$. It suffices to switch the signs of certain occurrences of the Artin generators in $V$. The corresponding "sign switching" algorithm is easy to derive from Corollary 6.1 and Proposition 5.2 of this paper.

2. Another method to simplify the procedure of computing $\mathbb{T}_{\phi}(\beta)$ is as follows. We take the smallest positive integer $k$ such that the braid $\beta^{k}$ is pure. Then we compute the values $\phi\left(\operatorname{REL}_{J}\left(\beta^{k}\right)\right)$ instead of $\phi\left(\operatorname{REL}_{J}\left(\beta^{n !}\right)\right)$. By property (2), the definition of pseudocharacters implies that

$$
\phi\left(\operatorname{REL}_{J}\left(\beta^{n !}\right)\right)=\frac{n !}{k} \cdot \phi\left(\operatorname{REL}_{J}\left(\beta^{k}\right)\right) .
$$

3.4. A remark on quasicharacters. If we get rid of the exponentiation of a braid and subsequent division by $n$ ! in formula $(*)$, then we obtain a formula converting pseudocharacters to quasimorphisms. The exact statement is as follows: we say that a map $R: B_{n} \rightarrow B_{n}$ is close to a map $Q: P_{n} \rightarrow P_{n}$ if for each finite subset $A \subset B_{n}$ the set

$$
\left\{(Q(\gamma))^{-1} \cdot R(\gamma \alpha): \gamma \in P_{n}, \alpha \in A\right\}
$$

is finite. Let $R_{J}: B_{n} \rightarrow B_{n}, J \subset\{1, \ldots, n\}$, be a family of maps that are close to the maps $\operatorname{REL}_{J}, J \subset\{1, \ldots, n\}$. (It is easy to construct such a family.) Then for each pseudocharacter $\phi \in \mathcal{P X}\left(B_{n}\right)$, the function $\widetilde{\mathbb{T}}_{\phi}: B_{n} \rightarrow \mathbb{R}$ defined by

$$
\widetilde{\mathbb{T}}_{\phi}(\beta):=\sum_{J \subset\{1, \ldots, n\}}(-1)^{|J|} \cdot \phi\left(R_{J}(\beta)\right)
$$

is a quasimorphism close to the pseudocharacter $\mathbb{T}_{\phi}$. In particular, the function $\widetilde{\mathbb{T}}_{\phi}-\mathbb{T}_{\phi}$ is bounded.

3.5. A remark on defects. To use a kernel pseudocharacter for the recognition of prime knots and links, it is necessary to know (at least an upper estimate for) its defect (see Theorem 1). There are several difficult problems related to the estimation of defects of pseudocharacters. Theorem 2 raises a natural problem of obtaining an upper bound for the defect $C_{\mathbb{T}(\phi)}$ of the kernel pseudocharacter $\mathbb{T}(\phi)$ in terms of the defect $C_{\phi}$ of a pseudocharacter $\phi \in \mathcal{P X}\left(B_{n}\right)$. It can be shown that

$$
C_{\mathbb{T}_{n}(\phi)} \leq\left(4 \cdot\left(2^{n}-C_{n}^{2}-C_{n}^{1}-C_{n}^{0}-C_{n}^{n}\right)+1\right) \cdot C_{\phi}=\left(2^{n+2}-2 n(n+1)-7\right) \cdot C_{\phi} \cdot
$$

If $n=3$, this yields $C_{\mathbb{T}_{3}(\phi)} \leq C_{\phi}$. (In fact, we easily check that the function $\mathbb{T}_{3}(\phi)-\phi$ is a homomorphism for each $\phi \in \mathcal{P X}\left(B_{3}\right)$, i.e., we have $C_{\mathbb{T}_{3}(\phi)}=C_{\phi}$.) However, we conjecture that, for $n>3$, the sharp upper bound for $C_{\mathbb{T}_{n}(\phi)}$ is considerably smaller than that presented above.

\section{§4. Pseudocharacters of normal subgroups of Finite index}

Let $G$ be an arbitrary group with a subgroup $F \subset G$. A pseudocharacter $\phi: F \rightarrow \mathbb{R}$ is said to be extendable to $G$ if there exists a pseudocharacter $\widetilde{\phi}: G \rightarrow \mathbb{R}$ whose restriction to $F$ coincides with $\phi$; the pseudocharacter $\widetilde{\phi}$ is called an extension of $\phi$. 
In $\S 8$, we encounter the problem of extendability of a pure braid group pseudocharacter to the braid group. We use the following two lemmas.

4.1. Lemma (the uniqueness of extension). Let $H$ be a normal subgroup of finite index $s$ in a group $G$. Let $\phi: H \rightarrow \mathbb{R}$ be a pseudocharacter. Assume that $\phi$ is extendable to $G$. Then $\phi$ has a unique extension $\widetilde{\phi}: G \rightarrow \mathbb{R}$. Moreover, for each $g \in G$ we have

$$
\widetilde{\phi}(g)=\frac{\phi\left(g^{s}\right)}{s} .
$$

Proof. This immediately follows from the definitions.

4.2. Lemma (a criterion for extendability). Let $H$ be a normal subgroup of finite index in a group $G$. Let $\phi: H \rightarrow \mathbb{R}$ be a pseudocharacter. Then $\phi$ is extendable to $G$ if and only if the following condition is fulfilled:

$$
\forall h \in H, g \in G: \phi\left(g h g^{-1}\right)=\phi(h) .
$$

Proof. First, observe that (4) is a necessary condition. Indeed, if $\phi$ has an extension $\widetilde{\phi}: G \rightarrow \mathbb{R}$, then, since each pseudocharacter is conjugacy invariant, for any $h \in H$ and $g \in G$ we have

$$
\phi\left(g h g^{-1}\right)=\widetilde{\phi}\left(g h g^{-1}\right)=\widetilde{\phi}(h)=\phi(h) .
$$

Now, we show that (4) is a sufficient condition. Suppose a pseudocharacter $\phi \in \mathcal{P X}(H)$ satisfies (4). Let $Q \subset G$ be a set of representatives of the cosets of $H$ in $G$ such that the class $1 \cdot H=H$ is represented by the trivial element 1 . Then each $g \in G$ is uniquely written in the form

$$
g=\mathbf{q}_{g} \mathbf{h}_{g}, \quad \text { where } \quad \mathbf{q}_{g} \in Q \quad \text { and } \quad \mathbf{h}_{g} \in H .
$$

Since the class $H \subset G$ is represented by the trivial element, for each $f \in H$ we have $\mathbf{q}_{f}=1$ and $\mathbf{h}_{f}=f$.

Let $\psi: G \rightarrow \mathbb{R}$ be the function defined by

$$
\psi(g):=\phi\left(\mathbf{h}_{g}\right) .
$$

We show that $\psi$ is a quasicharacter, i.e., that the following condition is fulfilled:

$$
\sup _{f, g \in G}|\psi(f g)-\psi(f)-\psi(g)|<\infty .
$$

Indeed, by the definition of $\psi$ we have

$$
\psi(f g)-\psi(f)-\psi(g)=\phi\left(\mathbf{h}_{f g}\right)-\phi\left(\mathbf{h}_{f}\right)-\phi\left(\mathbf{h}_{g}\right) .
$$

We see that

$$
\mathbf{h}_{f g}=\mathbf{q}_{f g}^{-1} f g=\mathbf{q}_{f g}^{-1} \mathbf{q}_{f} \mathbf{h}_{f} \mathbf{q}_{g} \mathbf{h}_{g} .
$$

This implies that the element $\mathbf{q}_{f g}^{-1} \mathbf{q}_{f} \mathbf{h}_{f} \mathbf{q}_{g}=\mathbf{h}_{f g} \mathbf{h}_{g}^{-1}$ is in $H$, and

$$
\phi\left(\mathbf{h}_{f g}\right)=\phi\left(\mathbf{q}_{f g}^{-1} \mathbf{q}_{f} \mathbf{h}_{f} \mathbf{q}_{g} \mathbf{h}_{g}\right)=\phi\left(\mathbf{q}_{f g}^{-1} \mathbf{q}_{f} \mathbf{h}_{f} \mathbf{q}_{g}\right)+\phi\left(\mathbf{h}_{g}\right)+\delta_{1},
$$

where $\left|\delta_{1}\right|$ does not exceed the defect $C_{\phi}$ of $\phi$.

Next, we see that the element $\mathbf{q}_{f g}^{-1} \mathbf{q}_{f} \mathbf{h}_{f} \mathbf{q}_{g}$ (which belongs to $H$ ) is conjugate (in $G$ ) to the element $\mathbf{q}_{g} \mathbf{q}_{f g}^{-1} \mathbf{q}_{f} \mathbf{h}_{f}$. Since condition (4) is assumed, it follows that

$$
\phi\left(\mathbf{q}_{f g}^{-1} \mathbf{q}_{f} \mathbf{h}_{f} \mathbf{q}_{g}\right)=\phi\left(\mathbf{q}_{g} \mathbf{q}_{f g}^{-1} \mathbf{q}_{f} \mathbf{h}_{f}\right)=\phi\left(\mathbf{q}_{g} \mathbf{q}_{f g}^{-1} \mathbf{q}_{f}\right)+\phi\left(\mathbf{h}_{f}\right)+\delta_{2},
$$

where $\left|\delta_{2}\right| \leq C_{\phi}$. Identities (6), (7), and (8) imply that

$$
\psi(f g)-\psi(f)-\psi(g)=\phi\left(\mathbf{q}_{g} \mathbf{q}_{f g}^{-1} \mathbf{q}_{f}\right)+\delta_{1}+\delta_{2} .
$$


We see that for any $g$ and $f$ the absolute value of $\phi\left(\mathbf{q}_{g} \mathbf{q}_{f g}^{-1} \mathbf{q}_{f}\right)$ does not exceed the constant

$$
C_{3}:=\max \left\{\left|\phi\left(a b^{-1} c\right)\right|: a, b, c \in Q\right\} .
$$

Thus,

$$
|\psi(f g)-\psi(f)-\psi(g)| \leq C_{3}+2 C_{\phi} .
$$

Clearly, this means that condition (5) is fulfilled, i.e., $\psi$ is a quasicharacter of $G$.

Condition (5) implies that for each element $g \in G$ the limit $\lim _{k \rightarrow \infty} \frac{\psi\left(g^{k}\right)}{k}$ exists, and the function $\bar{\psi}: G \rightarrow \mathbb{R}$ given by

$$
\bar{\psi}(g):=\lim _{k \rightarrow \infty} \frac{\psi\left(g^{k}\right)}{k}
$$

is a pseudocharacter (the proof can be found, e.g., in [11]).

We prove that the pseudocharacter $\bar{\psi} \in \mathcal{P X}(G)$ is an extension of $\phi$. For an arbitrary element $f \in H$ we have $\mathbf{h}_{f}=f$ (see the first part of the proof), whence $\psi(f)=\phi(f)$ by the definition of $\psi$. It follows that

$$
\bar{\psi}(f)=\lim _{k \rightarrow \infty} \frac{\psi\left(f^{k}\right)}{k}=\lim _{k \rightarrow \infty} \frac{\phi\left(f^{k}\right)}{k}=\lim _{k \rightarrow \infty} \frac{k \cdot \phi(f)}{k}=\lim _{k \rightarrow \infty}(\phi(f))=\phi(f) .
$$

Therefore, $\bar{\psi}$ is an extension of $\phi$. In particular, $\phi$ is extendable to $G$.

\section{§5. Strand Disentangling PROCEDURES FOR BRAids}

In this section, we introduce an auxiliary family of maps $\operatorname{rel}_{j}: B_{n} \rightarrow B_{n}(j=$ $1, \ldots, n)$. These maps are called strand disentangling procedures. They are defined in Proposition 5.2. These maps have a clear geometric meaning. The restriction of the map $\mathrm{rel}_{j}$ to the subgroup of pure braids is the homomorphism $\mathrm{REL}_{\{j\}} 3$ We use the procedures $\mathrm{rel}_{j}$ to prove properties of the homomorphisms REL $\mathrm{R}_{J}$. We note also that our definition of these procedures can be used in the computation of the braids $\operatorname{REL}_{J}(\beta)$ (see the remark about algorithms in $\S 3)$.

5.1. Notation. Let $\Sigma: B_{n} \rightarrow \Sigma_{n}$ be the epimorphism from the braid group to the symmetric group $\Sigma_{n}$ sending $\sigma_{i}$ to the permutation $(i, i+1)$. If $\beta \in B_{n}$ is a braid and $J \subset\{1, \ldots, n\}$ is a subset, we denote by $\beta(J)$ the image of $J$ under the permutation $\Sigma(\beta)$.

5.2. Proposition. Let $B_{n}$ be the braid group with Artin generators $\sigma_{1}, \ldots, \sigma_{n-1}$. Then there exists a unique family of $n$ maps

$$
\operatorname{rel}_{j}: B_{n} \rightarrow B_{n} \quad(j=1, \ldots, n)
$$

such that the following conditions are fulfilled:

For any $j \in\{1, \ldots, n\}, i \in\{1, \ldots, n-1\}, \delta \in\{+1,-1\}$, and $\alpha, \beta \in B_{n}$, we have

$$
\begin{gathered}
\operatorname{rel}_{j}\left(\sigma_{i}^{\delta}\right)=\left\{\begin{array}{cl}
\sigma_{i}^{-\delta} & \text { if } \sigma_{i}^{\delta}=\sigma_{j-1} \text { or } \sigma_{i}^{\delta}=\sigma_{j}^{-1}, \\
\sigma_{i}^{\delta} & \text { if } \sigma_{i}^{\delta} \notin\left\{\sigma_{j-1}, \sigma_{j}^{-1}\right\},
\end{array}\right. \\
\operatorname{rel}_{j}(\alpha \beta)=\operatorname{rel}_{\beta(j)}(\alpha) \cdot \operatorname{rel}_{j}(\beta) .
\end{gathered}
$$

5.3. Remark on geometric interpretation. The map $r e l_{j}$ has a clear geometric meaning. The transition from a braid $\beta$ to the braid $\operatorname{rel}_{j}(\beta)$ corresponds to the transformation of the geometric diagram $b$ representing $\beta$ that shifts the $j$ th strand of $b$ behind its other strands (the strand moves through other strands perpendicularly to the plane of the diagram). Figure 3 shows the diagrams for the braids $\sigma_{3} \sigma_{3} \sigma_{1} \sigma_{2}^{-1}$ and $\mathrm{rel}_{2}\left(\sigma_{3} \sigma_{3} \sigma_{1} \sigma_{2}^{-1}\right)=\sigma_{3}^{-1} \sigma_{3} \sigma_{1} \sigma_{2}=\sigma_{1} \sigma_{2}$ of $B_{4}$.

\footnotetext{
${ }^{3}$ The map $\mathrm{rel}_{j}$ is close to $\mathrm{REL}_{\{j\}}$ in the sense of the remark on quasicharacters in $\S 3$.
} 

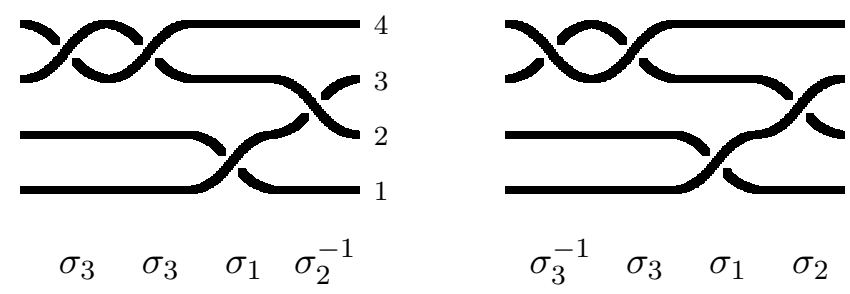

FIgURE 3. The diagrams $\mathcal{D}_{\sigma_{3} \sigma_{3} \sigma_{1} \sigma_{2}^{-1}}^{\langle 4\rangle}$ and $\mathcal{D}_{\sigma_{3}^{-1} \sigma_{3} \sigma_{1} \sigma_{2}}^{\langle 4\rangle}$.

Proof of Proposition 5.2. Existence. First, we show that there exists a family of maps satisfying the requirements of the proposition. We use the standard terminology of combinatorial group theory (see, e.g., 14). Let $A_{n}$ be the alphabet consisting of the symbols $\left(\sigma_{1}, \sigma_{1}^{-1}, \ldots, \sigma_{n-1}, \sigma_{n-1}^{-1}\right)$. Let $A_{n}^{*}$ be the set of all finite words over $A_{n}$. We denote by pr $: A_{n}^{*} \rightarrow B_{n}$ the natural surjective map that sends a word $w_{1} \ldots w_{s}$ to the braid $w_{1} \cdots w_{s}$. By definition, two words $V$ and $W$ in $A_{n}^{*}$ represent one and the same braid of $B_{n}$ if and only if $V$ and $W$ are related by a chain of the following elementary transformations:

i) deleting or inserting a subword of the form $\sigma_{i}^{\delta} \sigma_{i}^{-\delta}$;

ii) replacing a subword of the form $\sigma_{i} \sigma_{i+1} \sigma_{i}$ by $\sigma_{i+1} \sigma_{i} \sigma_{i+1}$ and vice versa;

iii) replacing a subword of the form $\sigma_{i} \sigma_{j}$ with $|i-j| \geq 2$ by $\sigma_{j} \sigma_{i}$.

Let the family of maps

$$
\operatorname{rel}_{j}^{*}: A_{n}^{*} \rightarrow A_{n}^{*} \quad(j=1, \ldots, n)
$$

be defined by the following conditions:

$$
\begin{aligned}
& \operatorname{rel}_{j}^{*}(\varnothing)=\varnothing ; \\
& \operatorname{rel}_{j}^{*}\left(\sigma_{i}^{\delta}\right)=\left\{\begin{array}{lll}
\sigma_{i}^{-\delta} & \text { if } \sigma_{i}^{\delta}=\sigma_{j-1} \text { or } \sigma_{i}^{\delta}=\sigma_{j}^{-1}, \\
\sigma_{i}^{\delta} & \text { if } & \sigma_{i}^{\delta} \notin\left\{\sigma_{j-1}, \sigma_{j}^{-1}\right\},
\end{array}\right. \\
& \quad \operatorname{rel}_{j}^{*}\left(w_{1} w_{2} \ldots w_{s}\right) \\
& \quad=\operatorname{rel}_{w_{2} \ldots w_{s}(j)}^{*}\left(w_{1}\right) \operatorname{rel}_{w_{3} \ldots w_{s}(j)}^{*}\left(w_{2}\right) \ldots \operatorname{rel}_{w_{s}(j)}^{*}\left(w_{s-1}\right) \operatorname{rel}_{j}^{*}\left(w_{s}\right) .
\end{aligned}
$$

(In (14), by the action of a word in $A_{n}^{*}$ on the set $\{1, \ldots, n\}$ we mean the action of the braid represented by that word; see Subsection 5.1.)

By considering all possibilities, we see that if two words $V$ and $W$ in $A_{n}^{*}$ are related by an elementary transformation, then the words $\operatorname{rel}_{j}^{*}(V)$ and $\operatorname{rel}_{j}^{*}(W)$ are related by a short chain of elementary transformations. This means that if two words $V$ and $W$ in $A_{n}^{*}$ represent one and the same braid, then the words $\operatorname{rel}_{j}^{*}(V)$ and $\operatorname{rel}_{j}^{*}(W)$ also represent one and the same braid. Consequently, the map $\operatorname{rel}_{j}^{*}: A_{n}^{*} \rightarrow A_{n}^{*}$ induces a certain map $\operatorname{rel}_{j}^{\prime}: B_{n} \rightarrow B_{n}$. Obviously, the form of equations (12), (13), and (14), which determine the maps rel ${ }_{j}^{*}$, implies that the family of maps $\operatorname{rel}_{j}^{\prime}: B_{n} \rightarrow B_{n}(j=1, \ldots, n)$ satisfies conditions (10) and (11). Thus, existence is proved.

Uniqueness. Assume that a family of maps $\operatorname{rel}_{j}^{\prime \prime}: B_{n} \rightarrow B_{n}(j=1, \ldots, n)$ satisfies conditions (10) and (11). Then, by induction on the length of a shortest word in $A_{n}^{*}$ representing a braid $\beta \in B_{n}$, we easily prove that the braids $\operatorname{rel}_{j}^{\prime \prime}(\beta)$ and $\operatorname{rel}_{j}^{\prime}(\beta)$ coincide for each braid $\beta \in B_{n}$. Consequently, the families $\operatorname{rel}_{j}^{\prime \prime}$ and $\operatorname{rel}_{j}^{\prime}$ coincide. 
5.4. Lemma. Let $i, j, k \in\{1, \ldots, n\}$, where $i<j$. Then

$$
\operatorname{rel}_{k}\left(a_{i j}\right)=\left\{\begin{array}{cl}
1 & \text { if } i=k \text { or } j=k, \\
a_{i j} & \text { if } k \notin\{i, j\} .
\end{array}\right.
$$

Proof. This easily follows from the definitions.

5.5. Lemma. For each $j \in\{1, \ldots, n\}$, the restriction $R_{j}:=\left.\operatorname{rel}_{j}\right|_{P_{n}}$ of the map $\mathrm{rel}_{j}$ : $B_{n} \rightarrow B_{n}$ to the subgroup $P_{n} \subset B_{n}$ of pure braids is a homomorphism from $P_{n}$ to $P_{n}$.

Proof. First, we prove that $R_{j}$ is a homomorphism. Let $\gamma_{1}$ and $\gamma_{2}$ be two arbitrary pure braids in $P_{n}$. Since each pure braid gives the identity permutation, we have $\gamma_{2}(j)=j$. By condition (11) of Proposition 5.2, we have

$$
R_{j}\left(\gamma_{1} \gamma_{2}\right)=\operatorname{rel}_{\gamma_{2}(j)}\left(\gamma_{1}\right) \cdot \operatorname{rel}_{j}\left(\gamma_{2}\right)=\operatorname{rel}_{j}\left(\gamma_{1}\right) \cdot \operatorname{rel}_{j}\left(\gamma_{2}\right)=R_{j}\left(\gamma_{1}\right) \cdot R_{j}\left(\gamma_{2}\right) .
$$

Now, it remains to use the definition of a homomorphism.

The fact that the image of $R_{j}$ lies in $P_{n}$ immediately follows from Lemma 5.4 (because the set of Markov generators generates $P_{n}$ ).

5.6. Lemma. For any $j \in\{1, \ldots, n\}$ and $\beta \in B_{n}$, the permutations $\Sigma(\beta)$ and $\Sigma\left(\operatorname{rel}_{j}(\beta)\right)$ coincide.

Proof. Let

$$
\beta=\sigma_{i_{1}}^{\delta_{1}} \cdots \sigma_{i_{s}}^{\delta_{s}}, \quad \text { where } \quad \sigma_{i_{k}} \in\left\{\sigma_{1}, \ldots, \sigma_{n-1}\right\} \quad \text { and } \quad \delta_{k} \in\{+1,-1\} .
$$

Then

$$
\Sigma(\beta)=\left(i_{1}, i_{1}+1\right) \circ \cdots \circ\left(i_{s}, i_{s}+1\right) .
$$

The conditions of Proposition 5.2 that determine the maps $\mathrm{rel}_{j}$ imply that the braid $\operatorname{rel}_{j}(\beta)$ equals the product $\sigma_{i_{1}}^{\delta_{1}^{\prime}} \cdots \sigma_{i_{s}}^{\delta_{s}^{\prime}}$ for some $\delta_{k}^{\prime} \in\{+1,-1\}$. Therefore,

$$
\Sigma\left(\operatorname{rel}_{j}(\beta)\right)=\left(i_{1}, i_{1}+1\right) \circ \cdots \circ\left(i_{s}, i_{s}+1\right)=\Sigma(\beta) .
$$

5.7. Lemma. Assume that two pure braids $\gamma_{1}$ and $\gamma_{2}$ in $P_{n} \subset B_{n}$ are conjugate in $B_{n}$ by a braid $\alpha$ :

$$
\gamma_{2}=\alpha \gamma_{1} \alpha^{-1}
$$

Let $j \in\{1, \ldots, n\}$. Then the pure braids $\mathrm{rel}_{j}\left(\gamma_{1}\right)$ and $\mathrm{rel}_{\alpha(j)}\left(\gamma_{2}\right)$ are conjugate in $B_{n}$ by the braid $\operatorname{rel}_{j}(\alpha)$ :

$$
\operatorname{rel}_{\alpha(j)}\left(\gamma_{2}\right)=\operatorname{rel}_{j}(\alpha) \cdot \operatorname{rel}_{j}\left(\gamma_{1}\right) \cdot\left(\operatorname{rel}_{j}(\alpha)\right)^{-1} .
$$

Proof. Relation (11) of Proposition 5.2 and the identity $\gamma_{2}=\alpha \gamma_{1} \alpha^{-1}$ imply

(15) $\operatorname{rel}_{\alpha(j)}\left(\gamma_{2}\right)=\operatorname{rel}_{\alpha(j)}\left(\alpha \gamma_{1} \alpha^{-1}\right)=\operatorname{rel}_{\gamma_{1} \alpha^{-1} \alpha(j)}(\alpha) \cdot \operatorname{rel}_{\alpha^{-1} \alpha(j)}\left(\gamma_{1}\right) \cdot \operatorname{rel}_{\alpha(j)}\left(\alpha^{-1}\right)$.

The permutations $\Sigma\left(\alpha^{-1} \alpha\right)$ and $\Sigma\left(\gamma_{1} \alpha^{-1} \alpha\right)$ are trivial, because the braid $\gamma_{1}$ is pure. This allows us to rewrite (15) in the following form:

$$
\operatorname{rel}_{\alpha(j)}\left(\gamma_{2}\right)=\operatorname{rel}_{j}(\alpha) \cdot \operatorname{rel}_{j}\left(\gamma_{1}\right) \cdot \operatorname{rel}_{\alpha(j)}\left(\alpha^{-1}\right) .
$$

Relation (11) of Proposition 5.2 and the identity $1=\alpha^{-1} \alpha$ imply that

$$
1=\operatorname{rel}_{j}(1)=\operatorname{rel}_{j}\left(\alpha^{-1} \alpha\right)=\operatorname{rel}_{\alpha(j)}\left(\alpha^{-1}\right) \cdot \operatorname{rel}_{j}(\alpha) .
$$

Therefore, $\operatorname{rel}_{\alpha(j)}\left(\alpha^{-1}\right)=\left(\operatorname{rel}_{j}(\alpha)\right)^{-1}$. This allows us to rewrite (16) in the form

$$
\operatorname{rel}_{\alpha(j)}\left(\gamma_{2}\right)=\operatorname{rel}_{j}(\alpha) \cdot \operatorname{rel}_{j}\left(\gamma_{1}\right) \cdot\left(\operatorname{rel}_{j}(\alpha)\right)^{-1},
$$

as required.

\footnotetext{
${ }^{4}$ By $a_{i j}$ we denote the Markov generators; see the definition in $§ 1$.
} 


\section{$\S 6$. THE HOMOMORPHISMS REL $J$}

In this section, we begin the study of the homomorphisms $\mathrm{REL}_{J}: P_{n} \rightarrow P_{n}, J \subset$ $\{1, \ldots, n\}$. First, we prove Proposition 3.1, where these homomorphisms are defined.

Proof of Proposition 3.1. Existence. Obviously, in the case where $J=\varnothing$, the trivial homomorphism satisfies condition (3). If $|J| \geq 1$, let $J=\left\{j_{1}, j_{2}, \ldots, j_{s}\right\}$, where $s:=|J|$. Let $R_{J}$ be the restriction of the composition $\operatorname{rel}_{j_{1}} \circ \mathrm{rel}_{j_{2}} \circ \cdots \circ \mathrm{rel}_{j_{s}}$ to the subgroup $P_{n}$ :

$$
R_{J}:=\left.\operatorname{rel}_{j_{1}} \circ \operatorname{rel}_{j_{2}} \circ \cdots \circ \mathrm{rel}_{j_{s}}\right|_{P_{n}} .
$$

We show that $R_{J}$ is a homomorphism satisfying (3). Indeed, by Lemma 5.5, for each $j \in\{1, \ldots, n\}$ the restriction $R_{j}:=\left.\operatorname{rel}_{j}\right|_{P_{n}}$ is a homomorphism from $P_{n}$ to $P_{n}$. This implies that

$$
R_{J}=\left.\mathrm{rel}_{j_{1}} \circ \mathrm{rel}_{j_{2}} \circ \cdots \circ \mathrm{rel}_{j_{s}}\right|_{P_{n}}=R_{j_{1}} \circ R_{j_{2}} \circ \cdots \circ R_{j_{s}} .
$$

Thus, the mapping $R_{J}$ is a homomorphism, being a composition of homomorphisms. The fact that the homomorphism $R_{J}$ satisfies (3) follows immediately from Lemma 5.4.

Uniqueness. The uniqueness statement is obvious, because the Markov generators generate $P_{n}$.

6.1. Corollary (from the proof of Proposition 3.1). Let $J=\left\{j_{1}, j_{2}, \ldots, j_{s}\right\}$ be a subset of $\{1, \ldots, n\}$. Then

$$
\mathrm{REL}_{J}=\left.\operatorname{rel}_{j_{1}} \circ \operatorname{rel}_{j_{2}} \circ \cdots \circ \operatorname{rel}_{j_{s}}\right|_{P_{n}} .
$$

6.2. Remark. The definition of the homomorphisms REL (see Proposition 3.1) shows immediately that for arbitrary subsets $I$ and $J$ of $\{1, \ldots, n\}$ we have

$$
\mathrm{REL}_{I \cup J}=\mathrm{REL}_{I} \circ \mathrm{REL}_{J} \text {. }
$$

6.3. Notation. Below we write $\mathrm{REL}_{j_{1}, \ldots, j_{s}}$ instead of $\mathrm{REL}_{\left\{j_{1}, \ldots, j_{s}\right\}}$.

6.4. Proposition. Suppose that two pure braids $\gamma_{1}$ and $\gamma_{2}$ in $P_{n} \subset B_{n}$ are conjugate in $B_{n}$ by a braid $\alpha$ :

$$
\gamma_{2}=\alpha \gamma_{1} \alpha^{-1}
$$

Then for each $J \subset\{1, \ldots, n\}$ the pure braids $\operatorname{REL}_{J}\left(\gamma_{1}\right)$ and $\operatorname{REL}_{\alpha(J)}\left(\gamma_{2}\right)$ are conjugate in $B_{n}$.

Proof. In the case where $J=\varnothing$, the proposition is proved by the following chain of identities:

$$
\operatorname{REL}_{\alpha(\varnothing)}\left(\gamma_{2}\right)=\operatorname{REL}_{\varnothing}\left(\gamma_{2}\right)=\gamma_{2}=\alpha \gamma_{1} \alpha^{-1}=\alpha \cdot \operatorname{REL}_{\varnothing}\left(\gamma_{1}\right) \cdot \alpha^{-1}
$$

If $J$ is a singleton (say $J=\{j\}$ ), then by Corollary 6.1 we have REL $J=\left.\mathrm{rel}_{j}\right|_{P_{n}}$. This implies our claim by Lemma 5.7 .

Finally, suppose that $|J| \geq 2$. Set $s:=|J|$ and $J=\left\{j_{s}, j_{s-1}, \ldots, j_{1}\right\}$. Then, by Corollary 6.1 and the formula $\gamma_{2}=\alpha \gamma_{1} \alpha^{-1}$, we have

$$
\begin{aligned}
\operatorname{REL}_{\alpha(J)}\left(\gamma_{2}\right) & =\operatorname{REL}_{\left\{\alpha\left(j_{s}\right), \ldots, \alpha\left(j_{1}\right)\right\}}\left(\alpha \gamma_{1} \alpha^{-1}\right) \\
& =\operatorname{rel}_{\alpha\left(j_{s}\right)} \circ \cdots \circ \operatorname{rel}_{\alpha\left(j_{1}\right)}\left(\alpha \gamma_{1} \alpha^{-1}\right) .
\end{aligned}
$$

By Lemma 5.7,

$$
\operatorname{rel}_{\alpha\left(j_{1}\right)}\left(\alpha \gamma_{1} \alpha^{-1}\right)=\operatorname{rel}_{j_{1}}(\alpha) \cdot \operatorname{REL}_{j_{1}}\left(\gamma_{1}\right) \cdot\left(\operatorname{rel}_{j_{1}}(\alpha)\right)^{-1} .
$$

Therefore,

$$
\operatorname{REL}_{\alpha(J)}\left(\gamma_{2}\right)=\operatorname{rel}_{\alpha\left(j_{s}\right)} \circ \cdots \circ \operatorname{rel}_{\alpha\left(j_{2}\right)}\left(\alpha_{2} \cdot \operatorname{REL}_{j_{1}}\left(\gamma_{1}\right) \cdot \alpha_{2}^{-1}\right),
$$

where $\alpha_{2}:=\operatorname{rel}_{j_{1}}(\alpha)$. 
By Lemma 5.6, we have $\alpha\left(j_{2}\right)=\alpha_{2}\left(j_{2}\right)$. From this, by Lemma 5.7 and Corollary 6.1, we deduce that

$$
\operatorname{rel}_{\alpha\left(j_{2}\right)}\left(\alpha_{2} \cdot \operatorname{REL}_{j_{1}}\left(\gamma_{1}\right) \cdot \alpha_{2}^{-1}\right)=\operatorname{rel}_{j_{2}}\left(\alpha_{2}\right) \cdot \operatorname{REL}_{j_{1}, j_{2}}\left(\gamma_{1}\right) \cdot\left(\operatorname{rel}_{j_{2}}\left(\alpha_{2}\right)\right)^{-1} .
$$

Thus,

$$
\operatorname{REL}_{\alpha(J)}\left(\gamma_{2}\right)=\operatorname{rel}_{\alpha\left(j_{s}\right)} \circ \cdots \circ \operatorname{rel}_{\alpha\left(j_{3}\right)}\left(\alpha_{3} \operatorname{REL}_{j_{1}, j_{2}}\left(\gamma_{1}\right) \alpha_{3}^{-1}\right),
$$

where $\alpha_{3}:=\operatorname{rel}_{j_{2}}\left(\alpha_{2}\right)$.

By Lemma 5.6, we have $\Sigma\left(\alpha_{3}\right)=\Sigma\left(\alpha_{2}\right)=\Sigma(\alpha)$. Continuing in the same way, at the sth step we obtain the following identity:

$\left(\mathrm{R}_{s+1}\right) \quad \operatorname{REL}_{\alpha(J)}\left(\gamma_{2}\right)=\alpha_{s+1} \cdot \mathrm{REL}_{\left\{j_{1}, j_{2}, \ldots, j_{s}\right\}=J}\left(\gamma_{1}\right) \cdot \alpha_{s+1}^{-1}$,

where $\alpha_{s+1}:=\operatorname{rel}_{j_{s}}\left(\alpha_{s}\right)=\operatorname{rel}_{j_{s}} \circ \operatorname{rel}_{j_{s-1}} \circ \cdots \circ \operatorname{rel}_{j_{1}}(\alpha)$.

\section{$\S 7$. The Alternating SUM LEMma}

7.1. Lemma. If a pure braid $\gamma \in P_{n}$ lies in the subgroup $\bar{P}_{n-1} \subset P_{n}$, then

$$
\sum_{J \subset\{1, \ldots, n\}}(-1)^{|J|} \cdot \operatorname{REL}_{J}(\gamma)=0 .
$$

(Here, the sum is considered in the group ring $\mathbb{Z} P_{n}$.)

Proof. We recall that the subgroup $\bar{P}_{n-1}$ is generated by the set $\left\{a_{i j}, 1 \leq i<j \leq n-1\right\}$ (see $\S 1$ ). Then the definition of the homomorphisms REL shows that the homomorphism $\mathrm{REL}_{n}$ is identical on $\bar{P}_{n-1}$. Furthermore, by Remark 6.2 we have $\mathrm{REL}_{I \cup\{n\}} \equiv \mathrm{REL}_{I} \circ \mathrm{REL}_{n}$ for each $I \subset\{1, \ldots, n-1\}$. Therefore, for any $\gamma \in \bar{P}_{n-1}$ and $I \subset\{1, \ldots, n-1\}$ we have

$$
\operatorname{REL}_{I \cup\{n\}}(\gamma)=\operatorname{REL}_{I} \circ \operatorname{REL}_{n}(\gamma)=\operatorname{REL}_{I}(\gamma) .
$$

Now we observe that

$$
\{J \mid J \subset\{1, \ldots, n\}\}=\bigcup_{I \subset\{1, \ldots, n-1\}}\{I, I \cup\{n\}\} .
$$

This implies the identity

$$
\sum_{J \subset\{1, \ldots, n\}}(-1)^{|J|} \cdot \operatorname{REL}_{J}(\gamma) \stackrel{(19)}{=} \sum_{I \subset\{1, \ldots, n-1\}}(-1)^{|I|} \cdot\left(\operatorname{REL}_{I}(\gamma)-\operatorname{REL}_{I \cup\{n\}}(\gamma)\right) \stackrel{(18)}{=} 0 .
$$

§8. ENDOMORPHISMS OF THE SPACE OF PSEUdochaRACTERS OF THE BRAID GROUP

8.1. Proposition. Let $\phi \in \mathcal{P X}\left(B_{n}\right)$ be a pseudocharacter, and let $k$ be a positive integer such that $k \leq n$. Let $\phi_{\langle k\rangle}: B_{n} \rightarrow \mathbb{R}$ be the function defined as follows:

$$
\phi_{\langle k\rangle}(\beta):=\sum_{J \in \mathbf{C}_{n}^{k}} \frac{\phi\left(\operatorname{REL}_{J}\left(\beta^{n !}\right)\right)}{n !},
$$

where

$$
\mathbf{C}_{n}^{k}:=\{I \subset\{1, \ldots, n\}:|I|=k\} .
$$

Then $\phi_{\langle k\rangle}$ is a pseudocharacter.

Proof. We consider the function $\mathcal{F}: P_{n} \rightarrow \mathbb{R}$ defined by

$$
\mathcal{F}(\gamma):=\sum_{J \in \mathbf{C}_{n}^{k}} \phi\left(\operatorname{REL}_{J}(\gamma)\right) .
$$

Since the composition of a homomorphism with a pseudocharacter is a pseudocharacter, it follows that for each $J$, the function $\gamma \mapsto \phi\left(\operatorname{REL}_{J}(\gamma)\right)$ is a pseudocharacter of $P_{n}$. 
Consequently, $\mathcal{F}$ is a sum of pseudocharacters, whence it is a pseudocharacter (of the pure braid group).

We prove that $\mathcal{F}$ is extendable to $B_{n}$ (see $\S 4$ for the definition). By Lemma 4.2, for this it suffices to show that for any braids $\beta \in B_{n}$ and $\gamma \in P_{n}$ we have

$$
\mathcal{F}\left(\beta \gamma \beta^{-1}\right)=\mathcal{F}(\gamma)
$$

By Proposition 6.4, for each $J \subset\{1, \ldots, n\}$ the braid $\operatorname{REL}_{J}(\gamma)$ is conjugate in $B_{n}$ to the braid $\operatorname{REL}_{\beta(J)}\left(\beta \gamma \beta^{-1}\right)$. Moreover, since $\phi$ is a pseudocharacter of $B_{n}$ (we recall that each pseudocharacter is conjugacy invariant), we have

$$
\phi\left(\operatorname{REL}_{J}(\gamma)\right)=\phi\left(\operatorname{REL}_{\beta(J)}\left(\beta \gamma \beta^{-1}\right)\right) .
$$

Now, by the definition of $\mathcal{F}$ we obtain

$$
\mathcal{F}(\gamma) \stackrel{\text { def }}{=} \sum_{J \in \mathbf{C}_{n}^{k}} \phi\left(\operatorname{REL}_{J}(\gamma)\right)=\sum_{J \in \mathbf{C}_{n}^{k}} \phi\left(\operatorname{REL}_{\beta(J)}\left(\beta \gamma \beta^{-1}\right)\right) .
$$

Next, we observe that each permutation of $\Sigma_{n}$ (in particular, the permutation $\Sigma(\beta)$ ) induces a bijection of the family $\mathbf{C}_{n}^{k}$. In other words, we have $\beta\left(\mathbf{C}_{n}^{k}\right)=\mathbf{C}_{n}^{k}$ (see Subsection 5.1). This implies that

$$
\sum_{J \in \mathbf{C}_{n}^{k}} \phi\left(\operatorname{REL}_{\beta(J)}\left(\beta \gamma \beta^{-1}\right)\right)=\sum_{I \in \beta\left(\mathbf{C}_{n}^{k}\right)} \phi\left(\operatorname{REL}_{I}\left(\beta \gamma \beta^{-1}\right)\right)=\mathcal{F}\left(\beta \gamma \beta^{-1}\right) .
$$

Combining (20) and (21), we get the required identity, $\mathcal{F}\left(\beta \gamma \beta^{-1}\right)=\mathcal{F}(\gamma)$, and by Lemma 4.2 this means that the pseudocharacter $\mathcal{F}$ is extendable onto $B_{n}$. By Lemma 4.1, the extension $\widetilde{\mathcal{F}}$ coincides with the function $\phi_{\langle k\rangle}$. Thus, $\phi_{\langle k\rangle}$ is a pseudocharacter, as required.

\section{§9. Proof of Theorem 2}

1a) First, we show that $\mathbb{T}_{\phi}$ is a pseudocharacter. Clearly, in the notation of Proposition 8.1 we have

$$
\mathbb{T}_{\phi}(\beta)=\sum_{k \in\{0, \ldots, n\}}\left((-1)^{k} \cdot \phi_{\langle k\rangle}(\beta)\right) .
$$

By Proposition 8.1, the functions $\phi_{\langle k\rangle}$ are pseudocharacters. Therefore, so is $\mathbb{T}_{\phi}$.

1b) Next, we prove that $\mathbb{T}_{\phi}$ is a kernel pseudocharacter. For this, it suffices to check that $\mathbb{T}_{\phi}$ sends the subgroup $\bar{B}_{n-1} \subset B_{n}$ to zero (see the definition in $\S 2$ ). Indeed, if $\alpha \in \bar{B}_{n-1}$, then the pure braid $\alpha^{n !}$ is in $\bar{B}_{n-1} \cap P_{n}=\bar{P}_{n-1}$, whence by Lemma 7.1 it follows that

$$
\mathbb{T}_{\phi}(\alpha)=\sum_{J \subset\{1, \ldots, n\}} \frac{(-1)^{|J|} \cdot \phi\left(\operatorname{rel}_{J}\left(\alpha^{n !}\right)\right)}{n !}=0 .
$$

2) Observe that by definition we have $\operatorname{REL}_{n}\left(P_{n}\right)=\bar{P}_{n-1}$ (see Subsection 1.2 and Proposition 3.1). Since the homomorphism $\Sigma: B_{n} \rightarrow \Sigma_{n}$ is surjective, for each $j \in$ $\{1, \ldots, n\}$ there is a braid $\alpha \in B_{n}$ such that $\alpha(j)=n$. Then, by Proposition 6.4 , the image $\operatorname{REL}_{j}\left(P_{n}\right)$ is conjugate in $B_{n}$ to the subgroup $\operatorname{REL}_{n}\left(P_{n}\right)=\bar{P}_{n-1}$. It follows (see Remark 6.2) that for each nonempty $I \subset\{1, \ldots, n\}$ the image $\operatorname{REL}_{I}\left(P_{n}\right)$ is conjugate in $B_{n}$ to a subgroup of $\bar{P}_{n-1}$.

If $\psi: B_{n} \rightarrow \mathbb{R}$ is a kernel pseudocharacter, then by definition we have

$$
\psi\left(\bar{P}_{n-1}\right) \subset \psi\left(\bar{B}_{n-1}\right)=\{0\} .
$$


Then the above arguments show that for each nonempty subset $I \subset\{1, \ldots, n\}$ we have $\psi\left(\operatorname{REL}_{I}\left(P_{n}\right)\right)=\{0\}$. In particular, for each $\beta \in B_{n}$ we have $\psi\left(\operatorname{REL}_{I}\left(P_{n}\right)\right)=0$. Therefore,

$$
\mathbb{T}_{\psi}(\beta)=\sum_{J \subset\{1, \ldots, n\}} \frac{(-1)^{|J|} \cdot \psi\left(\operatorname{REL}_{J}\left(\beta^{n !}\right)\right)}{n !}=\frac{(-1)^{|\varnothing|} \cdot \psi\left(\operatorname{REL}_{\varnothing}\left(\beta^{n !}\right)\right)}{n !}=\psi(\beta) .
$$

This means that $\mathbb{T}_{\psi}=\psi$.

The theorem is proved.

\section{REFERENCES}

[1] J. W. Alexander, A lemma on system of knotted curves, Proc. Nat. Acad. Sci. U.S.A. 9 (1923), 93-95.

[2] S. Baader, Asymptotic Rasmussen invariant, C. R. Math. Acad. Sci. Paris 345 (2007), 225-228. MR 2352924 (2008f:57012)

[3] M. Bestvina and K. Fujiwara, Bounded cohomology of subgroups of mapping class groups, Geom. Topol. 6 (2002), 69-89. MR1914565 (2003f:57003)

[4] J. S. Birman and W. W. Menasco, Studying links via closed braids. IV. Composite links and split links, Invent. Math. 102 (1990), 115-139. MR1069243 (92g:57010a)

[5] _ Erratum: "Studying links via closed braids. IV", Invent. Math. 160 (2005), no. 2, 447-452. MR $2138073(2005 \mathrm{~m}: 57006)$

[6] _ Studying links via closed braids. V. The unlink, Trans. Amer. Math. Soc. 329 (1992), no. 2, 585-606. MR1030509 (92g:57010b)

[7] P. Dehornoy, Braids and self-distributivity, Progr. Math., vol. 192, Birkhäuser, Basel, 2000. MR.1778150 (2001j:20057)

[8] J. Dyer and E. Grossman, The automorphism groups of the braid groups, Amer. J. Math. 103 (1981), no. 6, 1151-1169. MR0636956 (82m:20041)

[9] I. A. Dynnikov, Arc-presentations of links: monotonic simplification, Fund. Math. 190 (2006), 29-76. MR2232855(2007e:57006)

[10] I. V. Erovenko, On bounded cohomology of amalgamated products of groups, Int. J. Math. Math. Sci. 2004, no. 40, 2103-2121. MR.2100899 (2005j:20067)

[11] V. A. Faǔziev, The stability of the equation $f(x y)-f(x)-f(y)=0$, Acta Math. Univ. Comenian. (N.S.) 69 (2000), no. 1, 127-135. MR1796793(2003a:39026)

[12] J.-M. Gambaudo and É. Ghys, Braids and signatures, Bull. Soc. Math. France 133 (2005), no. 4, 541-579. MR 2233695 (2007d:57014)

[13] R. I. Grigorchuk, Some results on bounded cohomology, Combinatorial and Geometric Group Theory (Edinburgh, 1993), London Math. Soc. Lecture Note Ser., vol. 204, Cambridge Univ. Press, Cambridge, 1995, pp. 111-163. MR 1320279 (96j:20073)

[14] W. Magnus, A. Karrass, and D. Solitar, Combinatorial group theory: Presentations of groups in terms of generators and relations, Intersci. Publ., New York, 1966. MR0207802 (34:7617)

[15] A. V. Malyutin, Twist number of (closed) braids, Algebra i Analiz 16 (2004), no. 5, 59-91; English transl., St. Petersburg Math. J. 16 (2005), no. 5, 791-813. MR2106667 (2005j:57011)

[16] A. V. Malyutin and N. Yu. Netsvetaev, Dehornoy's ordering on the braid group and braid moves, Algebra i Analiz 15 (2003), no. 3, 170-187; English transl., St. Petersburg Math. J. 15 (2004), no. 3, 437-448. MR2052167 (2005b:20068)

[17] A. A. Markov, Foundations of the algebraic theory of braids, Trudy Mat. Inst. Steklov. 16 (1945), 3-53. (Russian) MR0017279 (8:131c)

St. Petersburg Branch, Steklov Institute of Mathematics, Russian Academy of Sciences, Fontanka 27, St. Petersburg 191023, Russia

E-mail address: malyutin@pdmi.ras.ru

Received 16/SEP/2008

Translated by THE AUTHOR 